\title{
Aa. Vv., Romans d'Antiquité et littérature du Nord. Mélanges offerts à Aimé Petit
}

\section{Maria Colombo Timelli}

\section{(2) OpenEdition}

1 Journals

\section{Edizione digitale}

URL: http://journals.openedition.org/studifrancesi/7809

DOI: 10.4000/studifrancesi.7809

ISSN: 2421-5856

\section{Editore}

Rosenberg \& Sellier

\section{Edizione cartacea}

Data di pubblicazione: 1 juillet 2009

Paginazione: 365-367

ISSN: 0039-2944

\section{Notizia bibliografica digitale}

Maria Colombo Timelli, «Aa. Vv., Romans d'Antiquité et littérature du Nord. Mélanges offerts à Aimé Petit», Studi Francesi [Online], 158 (LIII | II) | 2009, online dal 30 novembre 2015, consultato il 09 janvier 2021. URL: http://journals.openedition.org/studifrancesi/7809 ; DOI: https://doi.org/10.4000/ studifrancesi.7809

Questo documento è stato generato automaticamente il 9 janvier 2021.

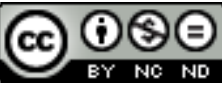

Studi Francesi è distribuita con Licenza Creative Commons Attribuzione - Non commerciale - Non opere derivate 4.0 Internazionale. 


\title{
Aa. Vv., Romans d'Antiquité et littérature du Nord. Mélanges offerts à Aimé Petit
}

\author{
Maria Colombo Timelli
}

\section{NOTIZIA}

SARAH BAUDELLE-MICHELS, MARIE-MADELEINE CASTELLANI, PHILIPPE LOGIÉ et EMMANUELLE POULAINGAUTRET éds., Romans d'Antiquité et littérature du Nord. Mélanges offerts à Aimé Petit, Paris, Honoré Champion, 2007.

1 La richesse de ce volume, tant par la quantité des contributions que par la diversité des objets d'investigation (malgré la présence de deux thèmes majeurs: les romans antiques et l'épopée), rend difficile la rédaction d'un compte rendu organique. On voudra donc bien nous pardonner la présentation décousue et par trop synthétique des notices qui suivent, qui ont pour but de fournir au moins le sujet des articles consacrés à la littérature française du Moyen Âge.

2 Sarah BAUDELLE-MICHELS (Renaut de Montauban bâtisseur, pp. 33-42) souligne l'importance et les enjeux du motif de la construction dans Renaut de Montauban, dont le protagoniste, fondateur de Montessor puis de Montauban, termine sa vie en manœuvre, attaché à la construction de l'Église Saint-Pierre à Cologne.

Dominique BOUTET (La récriture de Roncevaux dans la "Chronique rimée" de Philippe Mousket, pp. 55-65) voit dans l'œuvre de Philippe Mousket, plutôt qu'une simple récriture amplifiée du Pseudo-Turpin, une recomposition aux traits épiques et poétiques propres, dont l'auteur poursuit des principes esthétiques reconnaissables.

Annette BRASSEUR ("Les Trois Mots" de Guillaume le Clerc de Normandie, un Dit quelque peu publié, pp. 67-87) fournit la traduction en français moderne, accompagnée de notes, du Dit des Trois Mots qu'elle a édité critiquement dans «Bien dire et bien aprandre», 25, 2007, pp. 245-270 (voir SF 157, p. 150). 
5 Danielle BUSCHINGER (Les Filles-Fleurs dans l'“Alexandre de Paris", l'“Alexandre de Strasbourg" et le "Parsifal" de Richard Wagner, pp. 89-98) compare le traitement de cet épisode merveilleux dans les œuvres médiévales consacrées à Alexandre, pour conclure sur le syncrétisme opéré par Wagner, qui fond sa source principale, Wolfram von Eschenbach, et la rédaction de Strasbourg en s'inspirant du bouddhisme.

6 Marie-Madeleine castellani (Les larmes du crocodile et les dents de la mangouste, pp. 99-116) s'interroge sur les connaissances encyclopédiques de l'auteur de la version longue d'Athis et Prophilias, en particulier quant à quatre bêtes d'origine orientale: des bestelettes nommées aussi celidron, le cocodrille, et deux poissons non identifiés. Elle reconnaît des sources possibles dans le Physiologos, chez Isidore, sans doute chez Solin.

7 Caroline cazanave ("Huon de Bordeaux" à la Gaîté, en l'an IX de la République, pp. 117-135) présente un avatar peu connu de l'épopée du XIII ${ }^{\mathrm{e}}$ siècle: une pantomime féerie mise en scène au théâtre de la Gaîté en janvier 1801. Dérivée de l'Oberon de Wieland, malgré sa médiocrité littéraire, cette pièce constitue le premier Huon de Bordeaux théâtral conservé.

8 Denis collomp (Quand la femme se fait chevalier, ou comment concilier mesure féminine et démesure épique, pp. 137-157) souligne la nette séparation entre la femme-chevalier des chansons épiques et les femmes guerrières du roman antique; par ailleurs, la démesure semble marquer davantage les femmes-chevaliers du XIV ${ }^{e}$ que leurs devancières des $\mathrm{XII}^{\mathrm{e}}$ XIII ${ }^{\mathrm{e}}$ siècles.

9 Chantal CONNOCHIE-BOURGNE (Le roi aspergé ou les tours de fortune dans la seconde version de l'“Image du monde", pp. 169-185) étudie le remaniement transmis par le ms. de Londres, B.L. Harley 4333, chapitre V, où les métaphores de l'eau permettent d'interpréter l'existence des hommes et les événements de la vie.

10 Alain CORBELLARI (L'art narratif d'Henri de Valenciennes: de la "Chronique de l'Empereur Henri" au "Lai d'Aristote", pp. 187-197) compare ces deux œuvres, dont la seconde n'a été attribuée que récemment à Henri de Valenciennes, dans le but de préciser les liens entre les deux textes et de souligner les mérites d'un auteur qui «sait jouer de tous les registres de la littérature de son temps» (p. 197).

11 Raymond CORMIER (Valeurs païennes dans un roman d'Antiquité du XII siècle. Métempsycose vs. au-delà chrétien, pp. 199-216) s'interroge sur la théorie de la métempsycose, expliquée par Anchise à Enée tant dans l'Enéide que dans le Roman d'Eneas: à ses yeux, l'auteur médiéval a assimilé sans difficulté ces idées païennes sur la vie après la mort.

12 Nathalie COSSART-RENIERS (De la crosse au glaive, une problématique judiciaire dans "Doon de la Roche", pp. 217-234) relève les symétries entre deux épisodes, où un évêque ou un archevêque prennent les armes afin de rendre justice à une partie lésée; selon l'A. le choix de ces personnages au sein de l'idéologie féodale prouve l'originalité de cette chanson de geste.

13 Catherine CROIZY-NAQUET (D'une description l'autre, Carthage dans les manuscrits Bibl. Laurent., Florence, et BnF fr. 60, pp. 235-247) analyse les différences entre la description de Carthage dans la version 'ancienne' du Roman d'Enéas et celle du ms. fr. 60, révélatrice celle-ci d'une interprétation mise à jour au XIV ${ }^{e}$ siècle. d'Orange?, pp. 249-262) souligne le caractère singulier de cette chanson tardive, qui, 
tout en mettant en scène l'extinction du lignage de Guillaume, fonctionne comme un relais vers le premier Cycle de la Croisade.

Christine FERLAMPIN-ACHER (Le "Roman de Thèbes", geste de deux frères: le roman et son double, pp. 309-318) reconnaît dans la démultiplication des doubles conflictuels (fraternité, filiation, compagnonnage, relation amoureuse) un trait marquant de Thèbes, roman où la conciliation paraît impossible. En poussant plus loin son analyse, elle envisage dans le rapport entre le roman français et sa source latine une relation semblable à celle qui s'établit entre deux frères, et dans les conflits multiples «le symptôme d'un rapport probable du roman au monde et à ses sources» (p. 318).

Catherine GAULLIER-BOUGASSAS (La prise de Thèbes par Alexandre et les origines mythiques de la cité béotienne dans les "Romans d'Alexandre", pp. 331-342) compare en particulier l'œuvre de Thomas de Kent, qui justifie la destruction de la ville et souligne la sagesse du roi refusant les flatteries, et celle d'Alexandre de Paris, qui célèbre au contraire l'alliance entre le politique et les arts; pour les deux auteurs, Alexandre incarne dans cet épisode le bon exercice du pouvoir royal.

Gérard GROS (Estoire, escrit, livret. Étude sur le prologue et le préambule de l'“'Estoire del Saint Graal" (\$1-30), pp. 353-378) analyse finement le texte liminaire de l'Estoire: il reconnaît ainsi le rapport institué entre le livret offert au narrateur par Dieu lui-même, l'écriture confiée à l'homme et la vérité de l'estoire, garantie par la source.

Michèle GUÉRET-LAFERTÉ (Le discours de Sinon dans l'“Eneas": 'engin' et 'traïson', pp. 389-404) examine le discours de Sinon, enchâssé dans le récit qu'Enéas fait à Didon de la chute de Troie. Le remanieur médiéval manipule le modèle virgilien en accentuant la fonction persuasive de la parole.

19 Laurence HARF-LANCNER (Héroïsme et démesure: Alexandre et la Tour de Babel, pp. 405-422) suit le thème de la démesure et de l'homme révolté dans les versions médiévales de la vie d'Alexandre le Grand.

20 Jean-Charles HERBIN (La culture de l'auteur d'“Anseÿs de Gascogne”, pp. 423-435) dépouille les noms propres renvoyant à l'Antiquité grecque: malgré les lacunes et les flottements dans la tradition manuscrite, la culture hellénique de l'auteur anonyme s'avère significative.

21 Despina ION (Des parcours narratifs pour un drame familial: le projet d'écriture dans "Doon de la Roche", pp. 437-448) souligne la singularité de ce récit, fondé sur un drame familial qui se résout à travers les parcours particuliers des trois protagonistes Doon, Olive, Landris.

Danièle JAMES-RAoul (La poétique des noms de lieux dans l'“Eneas", pp. 449-464) constate que la gestion des toponymes dans Eneas, resserrés autour des villes de Troie, Cartage, Laurente, et occupant des places privilégiées dans les vers, participe de l'évolution de la fiction narrative vers le genre romanesque.

Huguette LEGROS (Alexandre dans le "Roman de Toute Chevalerie" de Thomas de Kent, un itinéraire spirituel des dieux à Dieu, pp. 515-529) montre comment Thomas de Kent renouvelle le personnage d'Alexandre, devenu un héros problématique qui parvient à la sagesse en suivant un parcours de conversion.

Philippe LogiÉ ("Floris et Lyriopé" de Robert de Blois ou le reflet d'Ovide, pp. 561-574): le roman de Robert de Blois oscille, dans le traitement du mythe de Narcisse, entre reprise 
du texte latin et liberté d'invention: la lecture personnelle de l'auteur se traduit en moralisation religieuse.

Jean-Pierre MARTIN (Les combats dans "Raoul de Cambrai", pp. 575-589) s'attache aux descriptions de combats: leur diversité répond certes à leur fonction narrative, mais confirme en même temps l'originalité d'une chanson de geste qui ne glorifie pas pour lui-même l'héroïsme guerrier.

Selon Laurence MATHEY-MAiLle (Peut-on parler d'anachronisme dans l'"Histoire des ducs de Normandie"?, pp. 591-599), l'anachronisme relève, tant chez Benoît de Sainte-Maure que chez Wace, d'un procédé conscient; sa fonction est double: pratique, puisqu'il répond à un effort de traduction, et idéologique, de transfert dans le passé des idéaux chevaleresques du XII ${ }^{\mathrm{e}}$ siècle.

Philippe MÉNARD (Deux fabliaux parallèles, "Gombert et les deux clercs" et le "Meunier et les deux clercs", pp. 601-611) mène une lecture comparative qui lui permet de mettre en relief constantes et variables et de conclure sur la postériorité du Meunier.

Francine MORA (Le "Conte de Floire et Blancheflor" peut-il être considéré comme un roman d'Antiquité?, pp. 613-625) relève quelques éléments qui rattachent Floire aux romans d'Antiquité, notamment l'onomastique et la pratique de l'ekphrasis; en même temps, l'auteur s'en sépare par son traitement du thème de la guerre et par sa conception de l'amour.

29 Aux yeux de Muriel отт (Glorïande, princesse infidèle?, pp. 627-642), Glorïande, conçue sur le modèle de la Sarrasine amoureuse d'un chevalier chrétien, s'en démarque par sa fidélité à son ami Karaeus.

Gianfelice PERON ('Ci faut li romans'. Topique et fonction de l'épilogue dans les romans français médiévaux, pp. 643-668): bien qu'exposés aux manipulations des copistes, les épilogues des œuvres médiévales sont porteurs d'informations souvent significatives (nom de l'auteur, du commanditaire, fonction attribuée à l'œuvre etc.) et contiennent des éléments topiques qui répondent à ceux du prologue.

31 Marylène POSSAMAï-PÉREZ (Ulysse ou le triomphe de la 'mètis', pp. 669-685) s'appuie sur le débat entre Ulysse et Ajax dans l'Ovide moralisé pour montrer comment la translatio opérée par l'auteur anonyme va dans le sens de l'exaltation des valeurs du XII siècle, entre autres la mètis individuelle.

32 Emmanuelle POULAIN-GAUTRET ('Mout a entre faire et dire': Rutebeuf et Guillaume de SaintAmour, pp. 687-698) s'interroge sur le 'je' poétique dans les deux poèmes que Rutebeuf a consacrés à Guillaume de Saint-Amour: apologiste d'un homme et d'une cause, le poète est en même temps en retrait et au premier plan, ce qui confirme la complexité du 'moi' poétique médiéval.

Claude RousSel ("Le Dit des annelets" de Jehan de Saint-Quentin, pp. 699-739) analyse la richesse intertextuelle de ce Dit, où se retrouvent de nombreux éléments d'origine folklorique et littéraire, hagiographique surtout; l'innovation la plus importante réside dans la présence des anneaux de fer, instrument de torture et de pénitence, qui n'est pas sans rapport avec la sexualité féminine.

Dans le cadre d'un projet en cours, le Thesaurus des motifs merveilleux, et sur la base de ses catégories, Jean-René vALETTE (Les motifs merveilleux à l'épreuve du Graal: l'exemple de la "Queste del Saint Graal", pp. 755-768) indexe les motifs graaliens dans la Queste (métalangage, thèmes récurrents, signes) visant à la dénomination topique. 
35 Jean-Claude VAlLECALLE (Roland sénateur de Rome dans "L'Entrée d'Espagne", pp. 769-779) reconnaît dans cette dénomination de Roland moins un anachronisme qu'une actualisation sur la Rome du XIV siècle et sur les valeurs du 'Trecento' italien. 